\title{
A STABILITY CONDITION FOR LINEAR COMPARISON SYSTEMS*
}

\author{
BY \\ R. W. GUNDERSON \\ Utah State University, Logan, Utah
}

Introduction. The following class of functions plays a particularly important role in the comparison theorem (vector Liapunov function) alternative to the classical theorems of the direct method of Liapunov.

Definition. A function $f: R^{n} \rightarrow R^{n}$ is said to be of type $W$ on a set $S \subset R^{n}$ if $f_{i}(a) \leq$ $f_{i}(b)(i=1,2, \cdots, n)$ for all $a, b$ in $S$ such that $a_{i}=b_{i}, a_{k} \leq b_{k}(i \neq k)$.

In the comparison theorems, one attempts to investigate the behavior of solutions to

$$
\dot{x}=f(t, x) \quad\left(x, f \in R^{n}\right)
$$

by constructing a system of first-order differential inequalities

$$
\dot{v}_{i}(t, x) \leq g_{i}(t, v(t, x)), \quad i=1,2, \cdots, m,
$$

satisfied by the vector Liapunov function $v=\left(v_{1}, v_{2} \cdots, v_{m}\right)$, where $g$ has the important restriction to being of type $W$. Providing $f, g$ and $v$ possess certain additional properties, it is then possible to conclude that the existence of a solution property (e.g. asymptotic stability) for the comparison system

$$
\dot{u}=g(t, u)
$$

implies the existence of the corresponding property for (1) (cf. [1]).

Linear Systems of Type $W$. Since very little is accomplished if the comparison system (3) cannot itself be analyzed, it follows that a most important class of comparison systems are those whose right sides are linear with constant coefficients. The following theorem provides a very useful and convenient condition for the stability of such systems:

TheOREM 1. Let the right side of the $n$th order constant coefficient linear system

$$
\dot{u}=A u, \quad A=\left\|a_{i k}\right\|_{1}^{n}
$$

be of type $W$ on $R^{n}$. Then (4) will be asymptotically stable if and only if

$$
a_{11}<0,\left|\begin{array}{ll}
a_{11} & a_{12} \\
a_{21} & a_{22}
\end{array}\right|>0, \cdots,(-1)^{n}\left|\begin{array}{llll}
a_{11} & a_{12} & \cdots & a_{1 n} \\
a_{21} & a_{22} & \cdots & a_{2 n} \\
a_{n 1} & a_{n 2} & \cdots & a_{n n}
\end{array}\right|>0 .
$$

Proof. The proof follows immediately from a result of Kotelyanskii [2, pp. 71-73]

* Received June 4, 1970. This work was supported by the National Aeronautics and Space Administration under Grant NGR-45-002-016. 
which gives the conditions (5) as a necessary and sufficient condition for all the characteristic values of a real matrix $A$ with nonnegative nondiagonal elements to have negative real parts. But it is easy to see that the right side of (4) will be of type $W$ if and only if $A$ satisfies the assumptions of Kotelyanskii's theorem.

As an illustration of the usefulness of Theorem 1, it is possible to provide the following short proof to a theorem given by Bailey in [3] which was established there using a comparatively lengthy root locus type argument. The result itself plays a central role in one of the few results concerned with providing a constructive procedure for obtaining the comparison equation.

TheOREM 2. The null solution of the system of linear differential equations

$$
\begin{gathered}
\dot{x}_{1}=-a_{1} x_{1}+b_{1} x_{n} \\
\dot{x}_{2}=-a_{2} x_{2}+b_{2} x_{1} \\
\vdots \\
\dot{x}_{n}=-a_{n} x_{n}+b_{n} x_{n-1}
\end{gathered}
$$

with $a_{i}$ and $b_{i}$ real, $a_{i}>0$ and $b_{i} \geq 0(i=1,2, \cdots, n)$, is asymptotically stable if and only if

$$
\prod_{i=1}^{n} \frac{b_{i}}{a_{i}}<1
$$

Proof. It is clear that the sign of the first $n-1$ principal diagonal minors of the matrix

$$
\left[\begin{array}{ccccc}
-a_{1} & 0 & \cdots & 0 & b_{1} \\
b_{2} & -a_{2} & \cdots & 0 & 0 \\
\cdot & \cdot & \cdot & \cdot & \cdot \\
0 & 0 & \cdots & b_{n} & -a_{n}
\end{array}\right]
$$

satisfy the conditions of Theorem 1 . The conditions of the theorem are satisfied then if and only if

$$
(-1)^{n}\left[\left(-a_{n}\right) \prod_{i=1}^{n-1}\left(-a_{i}\right)+(-1)^{n+1} b_{1} \prod_{i=2}^{n}\left(b_{i}\right)\right]=\prod_{i=1}^{n} a_{i}-\prod_{i=1}^{n} b_{i}>0
$$

that is, if and only if

$$
\prod_{i=1}^{n} \frac{b_{i}}{a_{i}}<1
$$

\section{REFERENCES}

[1] V. Lakshmikantham and S. Leela, Differential and integral inequalities, Vol. 1, Academic Press, New York, 1969

[2] F. R. Gantmacher, The theory of matrices, GITTL, Moscow, 1953; English transl., Vol. 2, Chelsea, New York, 1959

[3] F. N. Bailey, The application of Liapunov's second method to interconnected systems, J. Soc. Indust. Appl. Math. Ser. A Control 3, 443-462 (1966) 